\title{
Các nhân tố ảnh hưởng đến quyết định mua thực phẩm chức năng hỗ trọ̣ điều trị thoái hóa khớp của người tiêu dùng - Trường họ̣p tại tỉnh Đồng Tháp
}

\section{Factors affecting consumers' decision to purchase functional foods to support the treatment of osteoarthritis - The case in Dong Thap province}

\author{
Đặng Văn U't ${ }^{1 *}$, Lưu Tiến Thuận ${ }^{2}$ \\ ${ }^{1}$ Công ty cổ phần dược và vật tư y tế Bình Thuận, Việt Nam \\ ${ }^{2}$ Trường Đại học Cần Thơ, Việt Nam \\ *Tác giả liên hệ, Email: dangvanut@phapharco.com
}

THÔNG TIN

DOI: 10.46223/HCMCOUJS. econ.vi.15.3.1337.2020

Ngày nhận: 10/02/2020

Ngày nhận lại: 02/03/2020

Duyệt đăng: 05/03/2020

Tù khóa:

quyết định mua, thoái hóa khớp, thực phẩm chức năng

Keywords:

functional foods, osteoarthritis, purchase decision

TÓM TẮT

Mục tiêu của nghiên cứu là xác định và đo lường mức độ ảnh hưởng của các nhân tố đến quyết định mua thực phẩm chức năng hỗ trợ điều trị thoái khớp của người tiêu dùng tại tỉnh Đồng Tháp qua việc khảo sát 195 người tiêu dùng. Các phương pháp kiểm định được sử dụng trong nghiên cứu bao gồm: Hệ số tin cậy Cronbach's Alpha, phân tích nhân tố khám phá, và phân tích hồi quy. Kết quả nghiên cứu chỉ ra rằng có 6 nhân tố ảnh hưởng trực tiếp đến quyết định mua thực phẩm chức năng hỗ trợ điều trị thoái hóa khớp. Các nhân tố đó là: Truyền thông-quảng cáo; chuẩn chủ quan; chất lượng cảm nhận; thái độ chấp nhận thực phẩm chức năng hỗ trợ điều trị thoái hóa khớp; ý thức về sức khỏe; và sự an toàn khi dùng thực phẩm chức năng hỗ trợ điều trị thoái hóa khớp. Kết quả nghiên cứu đã có đóng góp về mặt học thuật trong việc thiết lập thang đo lường các nhân tố ảnh hưởng đến quyết định mua thực phẩm chức năng. Đồng thời, bài nghiên cứu đã đưa ra một số hàm ý quản trị nhằm gia tăng quyết định mua thực phẩm chức năng hỗ trợ điều trị thoái hóa khớp của người tiêu dùng.

ABSTRACT
The objective of the study is to identify and measure the
influence of factors on the decision to purchase functional foods to
support the treatment of osteoarthritis of consumers in Dong Thap
province by surveying 195 consumers. The methods used in the
study include: Cronbach's Alpha test, exploratory factor analysis,
and regression analysis. The results of the study show that there are
6 factors directly affecting the decision to purchase functional foods
to support the treatment of osteoarthritis. Factors include media-
advertising; subjective norms; perceived quality; acceptable attitude
of functional foods supporting the treatment of osteoarthritis; health
awareness; and the safety of taking supplements to support the
osteoarthritis treatment. The results of the study have contributed


academically to the creation of a measurement scale that affects the decision to purchase functional foods. At the same time, the research has proposed some administrative implications to increase the decision to purchase functional food to support the treatment of osteoarthritis of consumers.

\section{Tổng quan}

Cùng với xu hướng phát triển khoa học công nghệ, phát triển $\mathrm{y}$ - dược học, phát triển kinh tế,... chất lượng sống con người ngày một được nâng cao. Từ đó, việc chăm sóc sắc đẹp, chăm sóc sức khỏe nhằm tăng tuổi thọ ngày càng được quan tâm hơn. Việc tầm soát, phát hiện và điều trị kịp thời khi mắc bệnh, kể cả những bệnh hiểm nghèo là vô cùng quan trọng góp phần nâng cao chất lượng sống. Tuy nhiên, việc điều trị bệnh bằng dược phẩm như hiện nay không phải lúc nào cũng mang lại hiệu quả cao nhất. Đặc biệt, đối với những căn bệnh mạn tính thì việc điều trị phải mất nhiều thời gian, tốn nhiều chi phí và công sức, đồng thời khi điều trị thời gian dài sẽ phát sinh nhiều rủi ro vì tác dụng phụ của thuốc. Bệnh mạn tính thường gặp nhất hiện nay là thoái hóa khớp (THK). Việc điều trị THK bằng dược phẩm như hiện nay đang bộc lộ nhiều hạn chế vì tác dụng không mong muốn của chúng. Nếu dùng thời gian dài, không đủ liều và không đúng cách,... sẽ dễ dẫn đến nhiều rủi ro cho sức khỏe. Theo Bộ Y tế: Các thuốc chống viêm chỉ làm giảm các triệu chứng viêm mà không loại trừ được các nguyên nhân gây viêm, không làm thay đổi tiến triển của quá trình bệnh lý chính. Các thuốc này thường có tác dụng không mong muốn về tiêu hóa và tim mạch như: viêm, loét, thủng dạ dày-tá tràng, ruột non, suy tim sung huyết, bệnh lý mạch vành... Để hạn chế tác dụng phụ của dược phẩm, con người ngày nay có xu hướng dùng thực phẩm chức năng (TPCN) để thay thế. Theo thống kê của Hiệp hội TPCN Việt Nam thì số người sử dụng TPCN trong những năm qua không ngừng gia tăng. Cụ thể, nếu năm 2010, cả nước có khoảng 5,7 triệu người sử dụng, chiếm $6,6 \%$ dân số ở khắp 63 tỉnh, thành thì năm 2015 , số người dùng TPCN là 15,5 triệu người, chiếm $17 \%$ dân số ở khắp các tỉnh, thành và đến năm 2017 , số người dùng $\mathrm{TPCN}$ là gần 20 triệu người, chiếm $21 \%$ dân số. Hơn nữa, Cục An toàn thực phẩm, Bộ Y tế cho rằng: "Hiện nay, cả nước có khoảng 4.000 doanh nghiệp sản xuất kinh doanh TPCN". Rõ ràng tốc độ tăng trưởng của ngành hàng này và nhu cầu thị trường về TPCN là rất lớn, trong đó có dòng $\mathrm{TPCN}$ hỗ trợ điều trị THK.

Nhận thức được vai trò và công dụng của TPCN dùng để phòng và hỗ trợ điều trị bệnh THK, người tiêu dùng nói chung có xu hướng chọn TPCN hỗ trợ điều trị THK thay cho dược phẩm vì chúng ít tác dụng phụ. Trong những năm qua tại Đồng Tháp, người tiêu dùng cũng đã quan tâm đến dòng sản phẩm này. Song, việc nghiên cứu thị trường chưa được quan tâm đúng mức, do đó doanh thu vẫn còn hạn chế. Để hiểu sâu sắc hơn những nhân tố nào ảnh hưởng đến quyết định mua TPCN hỗ trợ điều trị THK của người tiêu dùng, nghiên cứu "Các nhân tố ảnh hưởng đến quyết định mua TPCN hỗ trợ điều trị THK của người tiêu dùng tại tỉnh Đồng Tháp" được thực hiện, từ đó đưa ra một số hàm ý quản trị giúp các doanh nghiệp đang sản xuất kinh doanh dòng sản phẩm này gia tăng quyết định mua nơi người tiêu dùng.

\section{Cơ sở lý luận và phương pháp nghiên cứu}

\subsection{Một số khái niệm}

Thực phẩm chức năng là thực phẩm hoặc sản phẩm dùng để hỗ trợ, phục hồi, duy trì hoặc tăng cường chức năng của các bộ phận trong cơ thể, có hoặc không tác dụng dinh dưỡng, tạo cho cơ thể tình trạng thoải mái, tăng sức đề kháng và giảm bớt nguy cơ bệnh tật. TPCN là giao thoa giữa thực phẩm và thuốc, giống thực phẩm về bản chất nhưng khác về hình thức, giống thuốc về hình thức nhưng khác về bản chất (D. Tran, 2018). 
Thoái hóa khớp: là hậu quả của quá trình cơ học và sinh học làm mất cân bằng giữa tổng hợp và huỷ hoại của sụn và xương dưới sụn. Sự mất cân bằng này có thể được bắt đầu bởi nhiều yếu tố: di truyền, phát triển, chuyển hoá và chấn thương, biểu hiện cuối cùng của thoái hóa khớp là các thay đổi hình thái, sinh hoá, phân tử và cơ sinh học của tế bào và chất cơ bản của sụn dẫn đến nhuyễn hoá, nứt loét và mất sụn khớp, xơ hoá xương dưới sụn, tạo gai xương và hốc xương dưới sụn (X. T. Nguyen, 2016).

Hành vi mua của người tiêu dùng: là toàn bộ hành động mà người tiêu dùng bộc lộ ra trong quá trình điều tra, mua sắm, sử dụng, đánh giá cho hàng hoá và dịch vụ nhằm thoả mãn nhu cầu của họ (D. M. Tran, 2013). Cũng có thể coi hành vi của người tiêu dùng là cách thức mà người tiêu dùng sẽ thực hiện để đưa ra quyết định sử dụng tài sản của mình (tiền bạc, thời gian, công sức...) liên quan đến việc mua sắm và sử dụng hàng hoá, dịch vụ nhằm thoả mãn nhu cầu cá nhân.

\subsection{Nhũng yếu tố cơ bản ảnh hưởng đến hành vi mua của người tiêu dùng}

Có nhiều nghiên cứu về hành vi mua của người tiêu dùng được thực hiện. Ajzen và Fishbein (1975) với Thuyết hành động hợp lý (TRA) đã đề xuất hai nhân tố ảnh hưởng đến ý định mua là thái độ và chuẩn chủ quan. Đến năm 1991, Ajzen đã phát triển TRA thành Lý thuyết hành vi hoạch định (TPB). Lý thuyết này đưa ra ba nhân tố quyết định đến hành vi mua gồm: chuẩn chủ quan, thái độ đối với hành vi, kiểm soát nhận thức hành vi. Đây là ba nhân tố cơ bản về hành vi mua của người tiêu dùng.

Dựa vào các lý trên, các tác giả kế thừa và ứng dụng vào các nghiên cứu của mình về $\mathrm{TPCN}$ ở nhiều nước khác nhau. Cụ thể, Christine Mitchel và Ring (2010) nghiên cứu thái độ và ý định mua TPCN của người tiêu dùng Thụy Điển. Kết quả cho thấy các yếu tố niềm tin và quy chuẩn tác động đến ý định mua thông qua hai nhân tố là thái độ và chuẩn chủ quan. Tương tự, O’Connor và White (2010) cho thấy có ba nhân tố của thuyết TPB là chuẩn chủ quan, thái độ và sự kiểm soát hành vi đều có tác động đến ý định mua TPCN. Annunziata và Vecchio (2010) nghiên cứu sự chấp nhận TPCN ở Italia. Nghiên cứu chỉ ra rằng các nhân tố cảm nhận về sức khỏe, sự tự tin, và sự thỏa mãn với sản phẩm có tác động đến sự chấp nhận TPCN của người tiêu dùng Italia. Ngoài ra, Markovina, Cacic, Kljusuric, và Kovacic (2011) đã chỉ ra rằng ý định mua TPCN ở người tiêu dùng Croatia bị tác động bởi các nhân tố là nhận thức về sức khỏe, sự tin tưởng vào TPCN, giá cả và chất lượng. Hơn nữa, nghiên cứu của Rezai, Teng, Mohamed, và Shamsudin (2012) ở Malaysia cho thấy có ba nhân tố tác động đến giới trẻ trong việc chọn mua TPCN gồm có sự kiểm soát hành vi, thái độ, nhận thức và cảm nhận của người tiêu dùng.

Trong nước, một số tác giả cũng đã nghiên cứu ý định mua TPCN của người tiêu dùng. Nghiên cứu của H. T. T. Nguyen (2015) về các yếu tố ảnh hưởng đến ý định mua TPCN ở Đà Nẵng bao gồm ý thức sức khoẻ, truyền thông, niềm tin kiểm soát, và nhận định của người tiêu dùng. P. H. N. Pham (2016) chỉ ra rằng ý định mua TPCN tại Vĩnh Long bị tác động bởi 3 nhân tố là thái độ đối với TPCN, sự kiểm soát hành vi được cảm nhận, chuẩn chủ quan. Riêng tác giả L. H. Nguyen (2016) phân tích các yếu tố ảnh hưởng đến quyết định mua dược phẩm tại Cần Thơ. Kết quả có 5 yếu tố tác động đến quyết định sử dụng thuốc nội là chất lượng, giá cả, yếu tố cảm quan, yếu tố kinh nghiệm và tính vị chủng. Nhìn chung, các nghiên cứu về quyết định mua TPCN còn giới hạn về số lượng, đa số các nghiên cứu chủ yếu tập trung vào ý định mua TPCN nói chung mà chưa đi sâu vào một loại $\mathrm{TPCN}$ nào cụ thể. Bài nghiên cứu này đã kế thừa các nhân tố ảnh hưởng và đồng thời nghiên cứu cụ thể trường hợp người tiêu dùng mua TPCN hỗ trợ điều trị THK.

\subsection{Mô hình nghiên cúu và giả thuyết nghiên cúu}

\subsubsection{Mô hình nghiên cúu}


Trên cơ sở lý thuyết, lược khảo các nghiên cứu trước đây, tham khảo ý kiến chuyên gia và phỏng vấn nhóm, tác giả đề xuất mô hình nghiên cứu như sau:

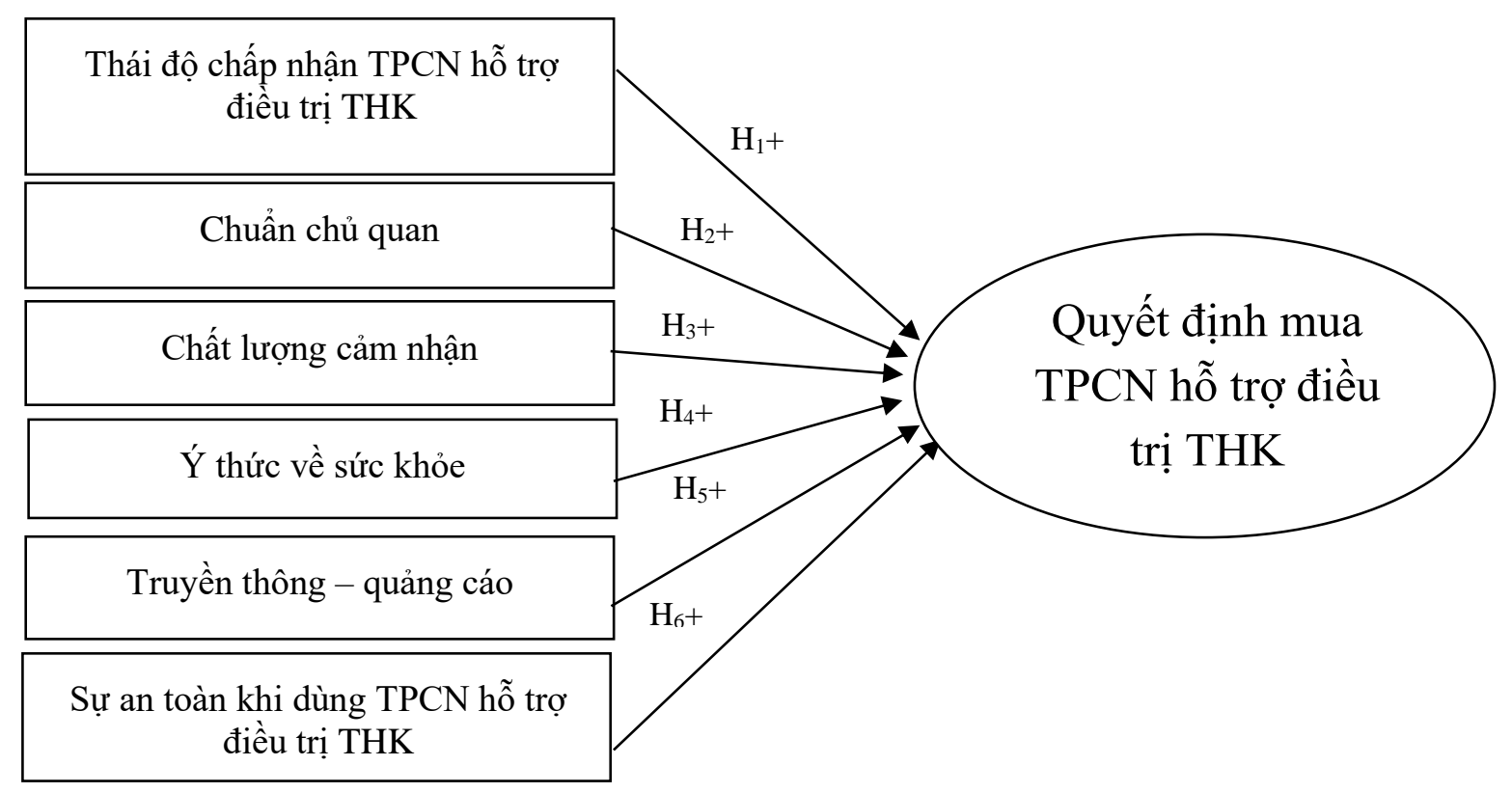

Hình 1. Mô hình nghiên cứu đề xuất các nhân tố ảnh hưởng đến quyết định mua TPCN hỗ trợ điều trị THK của người tiêu dùng tại tỉnh Đồng Tháp

\subsubsection{Giả thuyết nghiên cưu}

$H_{1}$ : Thái độ chấp nhận TPCN hỗ trọ điều trị THK có tác động đồng biến (+) đến quyết định mua TPCN hố trợ điều trị THK

$\mathrm{H}_{2}$ : Chuẩn chủ quan có tác động đồng biến (+) đến quyết định mua TPCN hỗ trợ điều trị THK

H3: Chất lương cảm nhận có tác động đồng biến (+) đến quyết định mua TPCN hỗ trợ điều tri $T H K$

H4: Ý thức về sức khỏe có tác động đồng biến (+) đến quyết định mua TPCN hỗ trợ điều tri $T H K$

$H_{5}$ : Truyền thông - quảng cáo có tác động đồng biến (+) đến quyết định mua TPCN hỗ trợ điều trị $T H K$

H6: Sự an toàn khi dùng TPCN hô̂ trợ điều trị THK có tác động đồng biến (+) đến quyêt định mua TPCN hố trọ điều trị THK

\subsection{Phương pháp nghiên cúu}

Bài nghiên cứu tiến hành phỏng vấn (03) chuyên gia là những giám sát bán hàng, giám đốc bán hàng có nhiều kinh nghiệm trong lĩnh vực TPCN, cùng nhóm bảy (07) thành viên đang bán dòng TPCN tại tỉnh Đồng Tháp. Nhóm đã thảo luận và sàng lọc các nhân tố ảnh hưởng trực tiếp đến quyết định mua TPCN hỗ trợ điều trị THK của người tiêu dùng tại tỉnh Đồng Tháp. Trên cơ sở đó, nghiên cứu định lượng được thực hiện với phương pháp chọn mẫu phi xác suất và chọn mẫu thuận tiện. Số mẫu phỏng vấn là 210 người tiêu dùng đã từng mua và dùng TPCN hỗ trợ điều trị THK (danh sách có được từ các nhà thuốc, các đại lý bán TPCN, các cơ sở đông y, hội y học cổ truyền, ...). Căn cứ vào mật độ dân cư cũng như thu nhập của người dân từng huyện, thị trong tỉnh 
Đồng Tháp, số lượng mẫu được phân chia theo từng khu vực như sau: thành phố Sa Đéc (70), thị xã Hồng Ngự (30), thành phố Cao Lãnh (70), huyện Tháp Mười (20) và huyện Lấp Vò (20). Sau khi kiểm tra bảng câu hỏi phỏng vấn, có 195 phiếu hợp lệ được phân tích trong nghiên cứu. Các phương pháp phân tích như kiểm định độ tin cậy Cronbach’s Alpha, phân tích nhân tố khám phá và mô hình hồi quy tuyến tính được sử dụng trong nghiên cứu nhằm tìm ra các nhân tố ảnh hưởng đến quyết định mua TPCN hỗ trợ điều trị THK của người tiêu dùng tại tỉnh Đồng Tháp.

\section{Kết quả nghiên cứu}

\subsection{Mô tả mẫu nghiên cứu}

Thống kê mô tả (Bảng 1) cho thấy, số nữ giới (63\%) cao hơn nam giới (37\%). Người cao tuổi dễ mắc bệnh THK (chiếm gần $72 \%$ đối với nhóm người có độ tuổi trên 50 ). Học vấn càng cao (Đại học và trên đại học), càng ít mắc bệnh (20\%). Bệnh THK xảy ra ở mọi đối tượng nghề nghiệp, tuy nhiên người nghỉ hưu, nhân viên văn phòng và công nhân mắc bệnh nhiều hơn (chiếm gần $70 \%$ ). Người có thu nhập từ 5 triệu trở lên chấp nhận mua TPCN nhiều hơn.

\section{Bảng 1}

Kết quả mô tả thông tin đáp viên

\begin{tabular}{|c|c|c|c|}
\hline & Mô tả & Số lượng & Tỉ lệ \% \\
\hline \multirow{2}{*}{ Giới tính } & Nam & 72 & 36,9 \\
\hline & Nữ & 123 & 63,1 \\
\hline \multirow{4}{*}{ Nhóm tuổi } & Dưới 40 tuổi & 9 & 4,6 \\
\hline & Từ 40-49 tuổi & 45 & 23,1 \\
\hline & Từ 50-59 tuổi & 70 & 35,9 \\
\hline & Trên 59 tuổi & 71 & 36,4 \\
\hline \multirow{4}{*}{ Trình độ học vấn } & Từ THPT trở xuống & 101 & 51,8 \\
\hline & Cao đẳng, THCN & 55 & 28,2 \\
\hline & Đại học & 35 & 17,9 \\
\hline & Trên đại học & 4 & 2,1 \\
\hline \multirow{6}{*}{ Nghề nghiệp } & Nhân viên văn phòng & 37 & 19,0 \\
\hline & Công nhân & 53 & 27,2 \\
\hline & Quản lý & 8 & 4,1 \\
\hline & Tiểu thương & 26 & 13,3 \\
\hline & Nghỉ hưu & 43 & 22,1 \\
\hline & Nghề khác & 28 & 14,4 \\
\hline \multirow{4}{*}{ Thu nhập } & Dưới 5 triệu & 20 & 10,3 \\
\hline & Từ 5 - 10 triệu & 70 & 35,9 \\
\hline & Từ 10 - 15 triệu & 84 & 43,1 \\
\hline & trên 15 triệu & 21 & 10,8 \\
\hline
\end{tabular}

Nguồn: Kết quả nghiên cứu 195 người tiêu dùng, 2020 


\section{2. Đánh giá độ tin cậy của thang đo bằng Hệ số Cronbach's Alpha}

Kết quả nghiên cứu ở (Bảng 2) cho thấy, tất cả các thang đo đều đạt chuẩn với hệ số Cronbach's Alpha đều lớn hơn 0,6 và dao động từ $0,763-0,885$. Tất cả các biến đều phù hợp và được sử dụng cho phân tích nhân tố khám phá.

\section{Bảng 2}

Kết quả phân tích độ tin cậy Cronbach's Alpha của các biến quan sát

\begin{tabular}{lrrrr}
\hline \multicolumn{1}{c}{$\begin{array}{c}\text { Biến } \\
\text { quan sát }\end{array}$} & $\begin{array}{c}\text { Trung bình thang } \\
\text { đo nếu loại biến }\end{array}$ & $\begin{array}{c}\text { Thang đo phương } \\
\text { sai nếu loại biến }\end{array}$ & $\begin{array}{c}\text { Hệ số tương quan } \\
\text { biến tô̂ng }\end{array}$ & $\begin{array}{c}\text { Cronbach's Alpha } \\
\text { nếu biến bị loại }\end{array}$ \\
\hline (1) Thái độ & & & Hệ số Cronbach's Alpha $=0,885$ \\
TD1 & 14,08 & 15,034 & 0,682 & 0,869 \\
TD2 & 13,82 & 15,179 & 0,670 & 0,872 \\
TD3 & 13,96 & 14,664 & 0,716 & 0,861 \\
TD4 & 14,05 & 14,652 & 0,749 & 0,854 \\
TD5 & 13,97 & 13,994 & 0,794 & 0,843 \\
\hline (2) Truyền thông & & & Hệ số Cronbach's Alpha $=0,776$ \\
TT1 & 6,31 & 1,845 & 0,603 & 0,710 \\
TT2 & 6,77 & 1,931 & 0,614 & 0,696 \\
TT3 & 6,69 & 1,998 & 0,622 & 0,689 \\
\hline (3) Chất lượng cảm nhận & & Hệ số Cronbach's Alpha & 0,769 \\
CL1 & 7,01 & 4,330 & 0,538 & 0,768 \\
CL2 & 6,62 & 4,113 & 0,656 & 0,628 \\
CL3 & 6,61 & 4,653 & 0,625 & 0,672 \\
\hline
\end{tabular}

(4) Ý thức về sức khỏe

\begin{tabular}{lllll} 
YT1 & 12,25 & 6,114 & 0,693 & 0,814 \\
YT2 & 12,12 & 5,933 & 0,634 & 0,828 \\
YT3 & 5,931 & 0,627 & 0,830 \\
YT4 & 12,15 & 5,942 & 0,621 & 0,832 \\
YT5 & 12,18 & 5,782 & 0,747 & 0,798 \\
\hline
\end{tabular}

(5) Sự an toàn

\begin{tabular}{lcccr} 
AT1 & 10,54 & 5,322 & 0,634 & 0,798 \\
AT2 & 10,57 & 5,555 & 0,637 & 0,796 \\
AT3 & 10,50 & 5,282 & 0,665 & 0,784 \\
AT4 & 10,49 & 5,169 & 0,700 & 0,767 \\
\hline (6) Chuẩn chủ quan & & & Hệ số Cronbach's Alpha $=0,844$ \\
CQ1 & 10,23 & 9,807 & 0,542 & 0,839
\end{tabular}




\begin{tabular}{lrrrr}
\hline $\begin{array}{c}\text { Biến } \\
\text { quan sát }\end{array}$ & $\begin{array}{c}\text { Trung bình thang } \\
\text { đo nếu loại biến }\end{array}$ & $\begin{array}{c}\text { Thang đo phương } \\
\text { sai nếu loại biến }\end{array}$ & $\begin{array}{c}\text { Hệ số tương quan } \\
\text { biến tổng }\end{array}$ & $\begin{array}{c}\text { Cronbach's Alpha } \\
\text { nếu biến bị loại }\end{array}$ \\
\hline CQ2 & 10,27 & 7,952 & 0,671 & 0,810 \\
CQ3 & 10,19 & 9,601 & 0,549 & 0,837 \\
CQ4 & 10,25 & 8,805 & 0,722 & 0,794 \\
CQ5 & 10,24 & 8,222 & 0,792 & 0,772 \\
\hline (7) Quyết định mua & & & Hệ số Cronbach's Alpha $=0,763$ \\
QD1 & 6,28 & 2,119 & 0,568 & 0.715 \\
QD2 & 6,51 & 2,035 & 0,644 & 0,623 \\
QD3 & 6,44 & 2,444 & 0,581 & 0,702 \\
\hline
\end{tabular}

Nguồn: Kết quả nghiên cứu 195 người tiêu dùng, 2020

\subsection{Phân tích nhân tố khám phá (EFA) các nhân tố ảnh hưởng đến quyết định mua TPCN hố trợ điều trị THK}

Kết quả phân tích EFA ở (Bảng 3) cho thấy tất cả các biến quan sát đều cho kết quả phù hợp với $\mathrm{KMO}=0,812(0,5 \leq \mathrm{KMO} \leq 1)$. Kiểm định Barlett's với giá trị $\mathrm{Sig}=0,00$ cho thấy các biến có tương quan với nhau trong tổng thể, tổng phương sai trích $=67,203$ giải thích được $67,2 \%$ biến thiên các biến quan sát. Sáu (06) nhóm nhân tố được trích tại trị số Eigenvalue =1,202 (>1).

\section{Bảng 3}

Kết quả phân tích trị số Eigenvalue, KMO và kiểm định Barlett’s

\begin{tabular}{|c|c|c|c|c|c|c|c|c|c|}
\hline \multirow{2}{*}{$\begin{array}{l}\text { Nhân } \\
\text { tố }\end{array}$} & \multicolumn{3}{|c|}{ Trị số Eigenvalue ban đầu } & \multicolumn{3}{|c|}{$\begin{array}{c}\text { Tổng bình phương hệ số } \\
\text { tải trích }\end{array}$} & \multicolumn{3}{|c|}{$\begin{array}{c}\text { Tổng bình phương hệ số } \\
\text { tải xoay }\end{array}$} \\
\hline & Tổng & $\begin{array}{c}\% \\
\text { phương } \\
\text { sai } \\
\end{array}$ & $\begin{array}{c}\text { Tích lũy } \\
\%\end{array}$ & Tổng & $\begin{array}{c}\% \\
\text { phương } \\
\text { sai } \\
\end{array}$ & $\begin{array}{c}\text { Tích lũy } \\
\%\end{array}$ & Tổng & $\begin{array}{c}\% \\
\text { phương } \\
\text { sai } \\
\end{array}$ & $\begin{array}{l}\text { Tích } \\
\text { lũy \% }\end{array}$ \\
\hline 1 & 5,657 & 22,628 & 22,628 & 5,657 & 22,628 & 22,628 & 3,482 & 13,926 & 13,926 \\
\hline 2 & 3,240 & 12,962 & 35,590 & 3,240 & 12,962 & 35,590 & 3,204 & 12,815 & 26,742 \\
\hline 3 & 2,752 & 11,008 & 46,598 & 2,752 & 11,008 & 46,598 & 3,194 & 12,777 & 39,519 \\
\hline 4 & 2,215 & 8,859 & 55,457 & 2,215 & 8,859 & 55,457 & 2,720 & 10,880 & 50,399 \\
\hline 5 & 1,735 & 6,939 & 62,396 & 1,735 & 6,939 & 62,396 & 2,132 & 8,527 & 58,926 \\
\hline 6 & 1,202 & 4,807 & 67,203 & 1,202 & 4,807 & 67,203 & 2,069 & 8,277 & 67,203 \\
\hline \multicolumn{4}{|c|}{ Trị số KMO } & & & & & & 0,812 \\
\hline \multicolumn{4}{|c|}{ Kiểm định Bartlett's } & Sig. & & & & & 0,000 \\
\hline
\end{tabular}

Nguồn: Kết quả nghiên cứu 195 người tiêu dùng, 2020

Ma trận xoay nhân tố (Bảng 4) cho kết quả có 25 biến quan sát gom thành 6 nhóm nhân tố, tất cả các biến quan sát đều có hệ số tải Factor Loading $>0,5$. 


\section{Bảng 4}

Kết quả ma trận xoay nhân tố

\section{Ma trận xoay nhân tố}

\begin{tabular}{llllllll}
\hline Các biến quan sát & \multicolumn{8}{c}{ Các nhân tố } \\
\cline { 2 - 8 } & 1 & 2 & 3 & 4 & 5 & 6 \\
\hline
\end{tabular}

TD5 Tôi rất tin tưởng khi dùng TPCN hỗ trợ điều trị THK.

0,876

TD4 Tôi rất an tâm khi dùng TPCN hỗ trợ điều trị THK.

0,816

TD3 Dùng TPCN hỗ trợ điều trị THK để phòng ngừa các bệnh về khớp.

0,796

TD1 Kết hợp TPCN hỗ trợ điều trị THK với dược phẩm sẽ giúp tôi mau hết bệnh.

0,794

TD2 Dùng TPCN hỗ trợ điều trị THK là rất cần thiết khi đau khớp.

0,731

YT5 Sức khỏe tôi phụ thuộc rất nhiều vào việc tôi tự chăm sóc.

YT1 Tôi có ý thức cá nhân về sức khỏe.

0,792

YT2 Tôi chú ý đến tình trạng sức khỏe tôi hàng ngày.

0,776

YT4 Tôi thấy cuộc sống ốm đau và bệnh tật là rất nghiêm trọng.

YT3 Tôi có trách nhiệm với sức khỏe của mình.

CQ5 Đồng nghiệp của tôi khuyên tôi nên dùng TPCN hỗ trợ điều trị THK.

CQ4 Các chuyên gia sức khỏe khuyên tôi nên dùng TPCN hỗ trợ điều trị THK.

CQ2 Những người thân của tôi đều dùng TPCN hỗ trợ điều trị THK.

CQ3 Nhân viên bán hàng khuyên tôi nên dùng TPCN hỗ trợ điều trị THK.

CQ1 Những người thân của tôi khuyên tôi nên dùng TPCN hỗ trợ điều trị THK.

AT4 TPCN hỗ trợ điều trị THK không nhiễm hóa chất độc hại.

AT3 TPCN hỗ trợ điều trị THK sẽ không gây độc khi dùng quá liều.

AT1 TPCN hỗ trợ điều trị THK rất an toàn khi sử dụng vì chúng có nguồn gốc hoàn toàn từ thiên nhiên. 


\begin{tabular}{|c|c|c|c|c|c|c|}
\hline \multicolumn{7}{|c|}{ Ma trận xoay nhân tố } \\
\hline \multirow{2}{*}{ Các biến quan sát } & \multicolumn{6}{|c|}{ Các nhân tố } \\
\hline & 1 & 2 & 3 & 4 & 5 & 6 \\
\hline $\begin{array}{l}\text { AT2 TPCN hỗ trợ điều trị THK rất ít tác } \\
\text { dụng phụ khi sử dụng. }\end{array}$ & & & & 0,764 & & \\
\hline $\begin{array}{l}\text { CL2 TPCN hỗ trợ điều trị THK rất công } \\
\text { hiệu. }\end{array}$ & & & & & 0,848 & \\
\hline $\begin{array}{l}\text { CL3 TPCN hỗ trợ điều trị THK giảm đau } \\
\text { nhanh sau khi uống. }\end{array}$ & & & & & 0,775 & \\
\hline $\begin{array}{l}\text { CL1 TPCN hỗ trợ điều trị THK trông đẹp } \\
\text { mắt và dễ uống. }\end{array}$ & & & & & 0,771 & \\
\hline $\begin{array}{l}\text { TT2 Nhà sản xuất khuyến khích tôi nên } \\
\text { dùng TPCN hỗ trợ điều trị THK. }\end{array}$ & & & & & & 0,808 \\
\hline $\begin{array}{l}\text { TT3 Đài phát thanh địa phương khuyến } \\
\text { khích tôi nên dùng TPCN hỗ trợ điều trị } \\
\text { THK. }\end{array}$ & & & & & & 0,763 \\
\hline $\begin{array}{l}\text { TT1 Quảng cáo khuyến khích tôi nên dùng } \\
\text { TPCN hỗ trợ điều trị THK. }\end{array}$ & & & & & & 0,758 \\
\hline
\end{tabular}

Nguồn: Kết quả nghiên cứu 195 người tiêu dùng, 2020

Tương tự, thực hiện phân tích EFA đối với biến phụ thuộc là Quyết định mua TPCN. Phân tích cho kết quả đạt yêu cầu với $\mathrm{KMO}=0,686$. Kiểm định Barlett's với giá trị $\mathrm{Sig}=0,00$, trị số Eigenvalue $=2,043$ và có 01 nhân tố (đặt tên Quyết định mua) được rút trích. Tổng phương sai trích $=68,084 \%$.

\subsection{Phân tích hồi quy đa biến}

Phân tích hồi quy đa biến được tiến hành bằng phương pháp "Enter”. Các biến QD: Quyết định mua TPCN hỗ trợ điều trị THK; TD: Thái độ chấp nhận TPCN hỗ trợ điều THK; TT: Truyền thông - quảng cáo; CL: Chất lượng cảm nhận; YT: Ý thức về sức khỏe; $\mathrm{AT}$ : Sự an toàn khi dùng TPCN hỗ trợ điều trị THK; và CQ: Chuẩn chủ quan được đưa vào cùng lúc. Kết quả phân tích hồi quy cho thấy, giá trị $\mathrm{R}^{2}$ của mô hình là 0,587 , giải thích được $58,7 \%$ biến thiên của các biến độc lập đến quyết định mua TPCN. Do đó, mức độ phù hợp của mô hình cũng được chấp nhận. Ngoài ra, hệ số Durbin-Watson $=2,163$, nằm trong khoảng 1,5 -2,5 nên không có hiện tượng tự tương quan. Giá trị $\operatorname{Sig}=0,00$ cho thấy mô hình hồi quy đa biến là phù hợp với tập dữ liệu. Hệ số phóng đại phương sai (VIF) của các biến đều nhỏ hơn 2 nên không có hiện tượng đa cộng tuyến. Các hệ số hồi quy đã chuẩn hóa đều dương và có giá trị $\mathrm{p}$-value nhỏ hơn 0,05 . Kết luận rằng: Các biến TD, TT, CL, YT, AT, CQ đều trực tiếp tác động đến quyết định mua TPCN hồ trợ điều trị THK (Bảng 5). Qua đó chấp nhận các giả thuyết từ $\mathrm{H}_{1}$ đến $\mathrm{H}_{6}$. 


\section{Bảng 5}

Kết quả phân tích hồi quy đa biến

\begin{tabular}{|c|c|c|c|c|c|c|c|c|}
\hline & \multirow[t]{2}{*}{ Biến } & \multicolumn{2}{|c|}{ Hệ số chưa chuẩn hóa } & \multirow{2}{*}{$\begin{array}{c}\begin{array}{c}\text { Hệ số đã } \\
\text { chuẩn hóa }\end{array} \\
\text { Beta }\end{array}$} & \multirow[t]{2}{*}{$\mathbf{T}$} & \multirow[t]{2}{*}{ Sig. } & \multicolumn{2}{|c|}{$\begin{array}{c}\text { Thống kê đa cộng } \\
\text { tuyến }\end{array}$} \\
\hline & & B & Sai số chuẩn & & & & Chấp nhận & VIF \\
\hline \multirow[t]{7}{*}{1} & (Constant) & $-0,661$ & 0,259 & & $-2,558$ & 0,011 & & \\
\hline & $\mathrm{TD}$ & 0,158 & 0,038 & 0,216 & 4,143 & 0,000 & 0,806 & 1,241 \\
\hline & TT & 0,320 & 0,058 & 0,299 & 5,542 & 0,000 & 0,753 & 1,328 \\
\hline & $\mathrm{CL}$ & 0,167 & 0,036 & 0,236 & 4,617 & 0,000 & 0,843 & 1,186 \\
\hline & YT & 0,241 & 0,060 & 0,204 & 4,048 & 0,000 & 0,861 & 1,162 \\
\hline & $\mathrm{AT}$ & 0,104 & 0,048 & 0,108 & 2,166 & 0,032 & 0,882 & 1,133 \\
\hline & CQ & 0,230 & 0,048 & 0,245 & 4,804 & 0,000 & 0,845 & 1,184 \\
\hline
\end{tabular}

Nguồn: Kết quả nghiên cứu 195 người tiêu dùng, 2020

Từ kết quả phân tích trên cho thấy, người tiêu dùng bị tác động rất lớn bởi truyền thông quảng cáo - TT, kế đến là chuẩn chủ quan - $\mathrm{CQ}$, chất lượng cảm nhận - $\mathrm{CL}$, thái độ chấp nhận TPCN hỗ trợ điều trị THK - TD, ý thức về sức khỏe - YT và sự an toàn khi dùng TPCN hỗ trợ điều trị THK - AT. Nghiên cứu cũng cho kết quả phù hợp với nghiên cứu của các tác giả Mitchel và Ring (2010), Annunziata và Vecchio (2010), O’Connor và White (2010), Markovina và cộng sự (2011), Rezai và cộng sự (2012), H. T. T. Nguyen (2015), P. H. N. Pham (2016), L. H. Nguyen (2016).

\section{Kết luận và hàm ý quản trị}

\subsection{Kết luận}

Qua việc khảo sát trực tiếp 195 người tiêu dùng về TPCN hỗ trợ điều trị THK tại tỉnh Đồng Tháp bằng phương pháp chọn mẫu phi xác suất. Bài nghiên cứu đã dựa trên cơ sở lý thuyết về Thuyết hành vi hoạch định $(\mathrm{TPB})$ và các nghiên cứu về hành vi tiêu dùng trước đây cũng như áp dụng các phương pháp phân tích như đánh giá độ tin cậy của thang đo, phân tích nhân tố khám phá, phân tích hồi quy đa biến để phân tích và đánh giá mức độ tác động của các nhân tố đến quyết định mua của người tiêu dùng. Kết quả nghiên cứu chỉ ra rằng có 06 nhân tố ảnh hưởng đến quyết định mua TPCN hỗ trợ điều trị THK của người tiêu dùng tỉnh Đồng Tháp với sự tác động từ cao đến thấp như sau: Truyền thông - quảng cáo, chuẩn chủ quan, chất lượng cảm nhận, thái độ chấp nhận đối với TPCN hỗ trợ điều trị THK, ý thức về sức khỏe, và sự an toàn khi dùng TPCN hỗ trợ điều trị THK. Bài nghiên cứu đã có những đóng góp nhất định về mặt học thuật trong việc thiết lập bộ thang đo lường các nhân tố ảnh hưởng đến quyết định mua TPCN hỗ trợ điều trị THK của người tiêu dùng. Trên cở sở đó, bài nghiên cứu đề xuất một số hàm ý quản trị mang tính thực tiễn cao như sau.

\subsection{Hàm ý quản trị}

Gia tăng truyền thông - quảng cáo: Các doanh nghiệp, các tổ chức đang kinh doanh, sản xuất dòng TPCN hỗ trợ điều trị THK cần tập trung vào kênh truyền thông - quảng cáo. Mục đích là cung cấp đầy đủ và chính xác các thông tin về công dụng và lợi ích của dòng TPCN hỗ trợ điều trị THK, đồng thời tuyên truyền vận động người dân nên dùng TPCN hỗ trợ điều trị THK. Điều này cũng phù hợp với quan điểm của Chủ tịch Hiệp hội TPCN Việt Nam (D. Tran, 2013), ông cho rằng “Công tác giáo dục truyền thông là hoạt động trung tâm, đi trước một bước trong tất cả các hoạt động nhằm phát triển TPCN ở Việt Nam”. 
Gia tăng chuẩn chủ quan: Cũng như truyền thông - quảng cáo, chuẩn chủ quan có tác động vô cùng mạnh mẽ trong quyết định mua TPCN hỗ trợ điều trị THK. Việc xây dựng một kênh bán hàng trực tiếp giữa những người có mối quan hệ thân thuộc trong gia đình, bạn bè hay đồng nghiệp sẽ giúp cho dòng sản phẩm TPCN hỗ trợ điều trị THK được dễ dàng đón nhận trong nhóm. Lý do là vì những người này luôn tin tưởng nhau và khi sử dụng thì sức lan tỏa càng lớn từ người này sang người khác. Để làm tốt điều này, doanh nghiệp cần có đội ngũ bán hàng rộng khắp, được đào tạo chuyên sâu về sản phẩm nhằm dễ dàng tiếp cận và thuyết phục họ mua và sử dụng.

Gia tăng chất lượng cảm nhận: Đây là nhân tố quyết định đến hành vi sau khi mua. Một sản phẩm có chất lượng thì người tiêu dùng mới hài lòng, tiếp tục sử dụng và trung thành với sản phẩm đó. Sự trung thành và tin tưởng sản phẩm của khách hàng không chỉ giới hạn ở chổ tiếp tục mua, mà họ còn khuyến khích, động viên cho người khác cùng sử dụng. Khi đó doanh nghiệp sẽ có thêm khách hàng, mở rộng thêm thị phần và bán được sản phẩm nhiều hơn. Để đạt mục tiêu về chất lượng, các doanh nghiệp cần tuân thủ các quy định, các tiêu chuẩn của Bộ Y tế về sản xuất kinh doanh TPCN, nâng cấp nhà máy đạt chuẩn GMP (Good Manufacturing Practices), ISO,...

Gia tăng thái độ chấp nhận TPCN hỗ trọ̣ điều trị THK: Các doanh nghiệp cần có chiến lược marketing tập trung vào nhân tố này nhằm nâng cao nhận thức, tạo niềm tin nơi người tiêu dùng, từ đó khách hàng sẽ có thái độ tích cực đối với các sản phẩm. Một trong những biện pháp nhằm gia tăng thái độ chấp nhận TPCN của người tiêu dùng là xây dựng chiến lược vận động, tuyên truyền và quảng cáo. Tuy nhiên, việc tạo thái độ tích cực nơi người tiêu dùng là không hề đơn giản và phải mất nhiều thời gian và chi phí. Do đó, việc vận động và tuyên truyền phải "đủ sâu", "đủ rộng" và “đủ liều” thì mới có kết quả tốt.

Gia tăng ý thức về sức khỏe: Các doanh nghiệp cần có chiến lược tác động đến ý thức của người tiêu dùng về sức khỏe của mình thông qua các chương trình có chuyên đề về sức khỏe, các buổi hội thảo chuyên sâu với nội dung ý thức, chăm sóc và bảo vệ sức khỏe. Từ đó, người tiêu dùng sẽ có ý thức, quan tâm, tìm kiếm và sử dụng dòng sản phẩm TPCN hỗ trợ điều trị THK không chỉ để điều trị khi mắc bệnh, mà còn để phòng bệnh THK.

Gia tăng sự an toàn khi dùng TPCN hỗ trọ̣ điều trị THK: Các doanh nghiệp cần công bố minh bạch thông tin về chuỗi cung ứng. Đó là thông tin từ khâu nuôi trồng, chế biến nguyên liệu đầu vào, khâu chọn nhà cung ứng tin cậy, các quy trình sản xuất theo các tiêu chuẩn, các quy định của Bộ Y tế như GMP, ISO, GAP cho đến bào quản, vận chuyển, phân phối (logistics)... để người tiêu dùng biết và tin tưởng vào sự an toàn của sản phẩm.

\subsection{Hạn chế và huớng nghiên cứu tiếp theo của đề tài}

Nghiên cứu không tránh khỏi những hạn chế nhất định: thứ nhất, nghiên cứu chỉ thực hiện trong phạm vi tỉnh Đồng Tháp nên tính khái quát hóa chưa cao; thứ hai, nghiên cứu thực hiện chung cho các sản phẩm thuộc nhóm TPCN hỗ trợ điều trị THK, chưa thực hiện được đối với tên một sản phẩm, một thương hiệu cụ thể. Đó cũng chính là những gợi ý cho việc mở rộng hướng nghiên cứu tiếp theo.

\section{Tài liệu tham khảo}

Ajzen, I. (1991). The theory of planned behavior. Organizational Behavior and Human Decision Processes, 50(2), 179-211.

Ajzen, I., \& Fishbein, M. (1975), Belief, attitude, intention and behaviour: An introduction to theory and research. Boston, MA: Addison-Wesley Publishing Co, Inc. 
Annunziata, A., \& Vecchio, R. (2010). Italian consumer attitudes toward products for well-being: The functional foods market. International Food and Argribusiness Management Review, 13(2), 19-50.

Hoang Trong, \& Chu, N. N. M. (2008). Phân tích dũ liệu nghiên cúu với SPSS [Analyze research data with SPSS]. Ho Chi Minh, Vietnam: Nhà xuất bản Hồng Đức.

Linh, T. (2018). "Giải nguy” cho 3.000 doanh nghiệp sản xuất TPCN có nguy cơ đóng của? ["Rescue" for 3,000 industrial food production enterprises in danger of closing?]. Retrieved January 16, 2020, from https://laodong.vn/y-te/giai-nguy-cho-3000-doanhnghiep-san-xuat-tpcn-co-nguy-co-dong-cua-632991.ldo

Markovina, J., Cacic, J., Kljusuric, J. G., \& Kovacic, D. (2011), Young consumers' perception of functional foods in Croatia. British Food Journal, 113(1), 7-16.

Mitchel, C., \& Ring, E. (2010). Swedish consumers' attitudes and purchase intentions of unctional food: A study based on the theory of planned behavior (Master's thesis). Umea School of Business, Sweden.

Nguyen, H. T. T. (2015). Nghiên cứu các yếu tố ảnh hương đến ý định mua TPCN của người tiêu dùng Đà Nã̃ng [Research on factors affecting consumers' intention to buy industrial bonds in Da Nang] (Master's thesis). The University of Danang, Vietnam.

Nguyen, L. H. (2016). Phân tích các yếu tố ảnh hưởng đến quyết định mua dược phẩm của người tiêu dùng tại thành phố Cần Tho [Analysis of factors affecting consumers' decisions to buy pharmaceuticals in Can Tho city] (Master's thesis). Can tho University, Vietnam.

Nguyen, X. T. (2016). Hướng dẫn chẩn đoán và điều trị các bệnh cơ xưong khớp [Guidelines for the diagnosis and treatment of musculoskeletal diseases]. Hanoi, Vietnam: Nhà xuất bản Y học.

O’Connor, E. L., \& White, K. M. (2010). Willingness to trial functional foods and vitamin function food: The role of attitudes, subjective norms and dread of risks. Food Quality and Preference, 21(1), 75-81.

Pham, P. H. N. (2016). Nghiên cúu các yếu tố ảnh hương đến ý định mua TPCN của người tiêu dùng tại tỉnh Vinh Long [Research on factors affecting consumers' intention to buy industrial bonds in Vinh Long province] (Master's thesis). Mekong University, Vietnam.

Rezai, G., Teng, P. K., Mohamed, Z., \& Shamsudin, M. N. (2012). Functional food knowledge and perceptions among young consumers in Malaysia. International Journal of Economics and Management Sciences, 6(3), 307-312.

Thuy Giang (2018). Sự phát triển "thần tốc" của thị trường thực phẩm chức năng [The rapid development of the functional food market]. Retrieved January 16, 2020, from http://tuyengiao.vn/y-te-cong-dong/su-phat-trien-than-toc-cua-thi-truong-thuc-pham-chucnang-117594

Tran, D. (2013). Chiến lược phát triển thực phẩm chức năng giai đoạn 2013-2020 và tầm nhìn 2030 [Strategy for functional food development for the period 2013-2020 and vision to 2030]. Retrieved December 16, 2019, from Hiệp hội thực phẩm chức năng Việt Nam website: http://www.vaff.org.vn/tin-tuc-44/tin-hoat-dong-45/chien-luoc-phat-trien-thucpham-chuc-nang-giai-doan-2013-2020-b3418

Tran, D. (2018). Thuật ngũ an toàn thực phẩm [Food safety term]. Hanoi, Vietnam: Nhà xuất bản Y học.

Tran, D. M. (2013). Giáo trình Marketing căn bản [Basic marketing curriculum]. Hanoi, Vietnam: Nhà xuất bản Kinh tế quốc dân. 